merkenswerterweise erniedrigt sich auch die Frequenz. an der die jeweiligen Maxima der Schallspannung auftreten, obwohl man rein mechanisch eine Erhöhung der Resonanzfrequenz erwarten würde. Hierïur ist der Druckverlust, den der durch die Operation ausgelöste Blutverlust hervorruft, verantwortlich (Verminderung der Einspannung der Basalplatte des Flagellum im Pedicellus ${ }^{6}$ ).

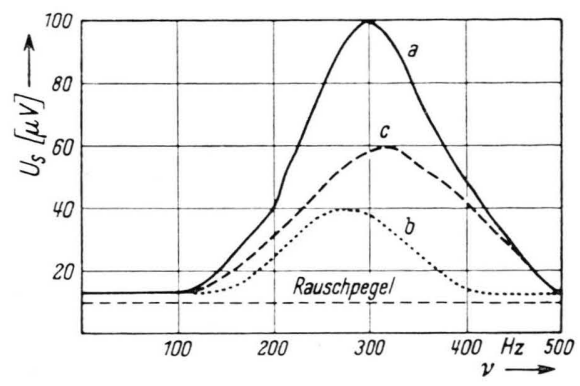

Abb. 2. Schallspannung $U_{s}$ in Funktion der Frequenz $v$. d) Der Frequenzbereich, in dem die Tiere hören können, ergibt sich aus den Messungen im Schallfeld entsprechend dem früheren Befund ${ }^{1}$ zu 100 bis $500 \mathrm{~Hz}$. (Rотн gab als obere Grenze $750 \mathrm{~Hz}$ an, was nach meinen Messungen zu hoch ist.)

e) Durch Vergleich mit dem früheren Ergebnis ${ }^{1}$. wonach bei mechanischer Erregung die Schallspannungs-Amplitude bei konstanter Antennenelongation unabhängig von der Frequenz ist, ergibt sich, daß der charakteristische Frequenzgang der Schallspannungs-Amplitude (Abb. 2, Kurve a) allein bedingt ist durch das Resonanzverhalten der Antenne, daß das Johnston-Organ selbst zwischen 100 und $500 \mathrm{~Hz}$ frequenzunabhängig arbeitet.

Die Untersuchungen wurden im Institut für Schwingungsforschung, Tübingen, durchgeführt. Ich danke Herrn Prof. Dr. H. Tischner für die Bereitstellung der Mittel sowie für zahlreiche, anregende Diskussionen.

\title{
Über die Verteilung von Radioyttrium und radioaktiven Seltenen Erden im Säugerorganismus
}

\author{
I.: Verteilungsversuche mit trägerfreiem Radioyttrium $\left({ }^{91} \mathrm{Y}\right)$ an Meerschweinchen \\ Von Ernst Spode
}

\begin{abstract}
Aus dem Institut für Medizin und Biologie der Deutschen Akademie der Wissenschaften zu BerlinBuch (Präsident: Prof. Dr. Dr. h. c. W. FrIedrich), Arbeitsbereich Angewandte Isotopenforschung (Z. Naturforschg. 13 b, 286-291 [1958] ; eingegangen am 27. Dezember 1957)
\end{abstract}

\begin{abstract}
Es wird über das Verteilungsmuster von Radioyttrium $\left.{ }^{91} \mathrm{Y}\right)$ in Meerschweinchen nach intrakardialer, intraperitonealer und subkutaner Applikationsweise und die dabei auftretenden Unterschiede berichtet. Nach der Inkorporation von Yttrium muß mit einer Leberpassage vor der Knochenspeicherung gerechnet werden. Die hohen Aktivitäten in Leber und Nieren bedürfen mehr als bisher der strahlenbiologischen Beachtung.
\end{abstract}

Das Interesse für die radioaktiven Yttriumisotope ${ }^{90} \mathrm{Y}$ und ${ }^{91} \mathrm{Y}$ ist im Laufe der Zeit ständig gestiegen. Die Gründe hierfür liegen einmal in der Tatsache. daß ${ }^{90} \mathrm{Y}$, Folgeprodukt von ${ }^{90} \mathrm{Sr}$, in der Therapie eine gewisse Rolle zu spielen beginnt, teils als ge. schlossenes Präparat zur Kontaktbestrahlung, teils in kolloidaler Form für intrakavitäre Anwendung. Zum anderen tritt ${ }^{91} \mathrm{Y}$ als Spaltprodukt bei der Kern-

1 E. L. Dobson, J. W. Gofmax, H. B. Jones, L. S. Kelly u. I. A. Walker, J. Labor. clin. Med. 34, 305 [1949].

2 J. Schubert, J. Labor. clin. Med. 34, 313 [1949].

3 J. Schubert u. M. R. White, J. biol. Chemistry 184, 191 [1950].

4 J. Schubert, M. P. Finkel, M. R. White U. G. M. Hirsch, J. biol. Chemistry 182, 635 [1950].

5 B. P. Kidman, M. L. Tutt u. J. M. Vaughan, a) J. Pathol. Bacteriol. 62, 209 [1950]; b) J. Pathol. Bacteriol. 63, 253 [1951]; c) Nature [London] 167, 858 [1951]. spaltung in größerer Menge auf und muß wegen seiner Langlebigkeit und seines biologischen Verhaltens zu den besonders gefährlichen Radioisotopen gerechnet werden. Untersuchungen über die Verteilung des Radioyttriums im Versuchstier sind daher mit beiden Isotopen schon mehrfach durchgeführt worden ${ }^{1-8}$. Allerdings wurden dabei meist nur die wesentlichsten Organe (z. B. Leber, Milz, Knochen)

\footnotetext{
6 N. S. McDonald, R. E. Nusbaum, G. V. Alexander. F. Ezirliam, P. Spain u. D. E. Rounds, J. biol. Chemistry 195, 837 [1952].

7 R. Lewin. H. E. Hart, J. Greenberg, H. Spencer, K. G. Stern u. D. Laszlo, a) Proc. Radioisotope Conf. 1, 125 [1954]; b) Science [Washington] 119, 880 [1954].

8 G. A. Andrews, G. C. Kyker, R. M. Kniseley u. E. L. Palmer, Proc. Amer. Assoc. Cancer Res. 2, 46 [1955].
} 
sowie nur einzelne Untersuchungszeitpunkte nach erfolgter Inkorporation berücksichtigt, so daß ein vollständiges Bild bislang vermißt wird. In letzter Zeit haben sich besonders Graul und Hundeshagen ${ }^{9}$. Kawin ${ }^{10}$, Scheer ${ }^{11}$ und Laszlo et coll. ${ }^{12}$ mit Fragen der Yttriumverteilung und -ausscheidung, meist vom Standpunkt des Strahlenschutzes und therapeutischer Erwägungen aus, beschäftigt. Auch C $\mathrm{ATSCH}$ setzte sich in größerem Rahmen mit dem Problem der Yttriuminkorporation auseinander ${ }^{13 \mathrm{a}, \mathrm{b}}$.

Aus den Untersuchungen geht hervor, daß Radioyttrium - in Abhängigkeit von der inkorporierten Gewichtsdosis - vor allem als Knochensucher anzusprechen ist, wobei die Ablagerung vorwiegend im Bereich des Periosts und Endosts unter besonderer Berücksichtigung der Epiphysen zu erfolgen scheint ${ }^{14-17}$. Unter Umständen jedoch muß auch mit einer beträchtlichen Leberspeicherung gerechnet wer. den. Die eingehenden Versuche von Graul und Hundeshagen ${ }^{9}$ ergaben zudem eine starke Anreicherung des Yttriums in Pankreas, Darmtrakt und Hoden (intraperitoneale Injektion). Eigene Untersuchungen weisen darauf hin, daß die Applikationsart offenbar für das Verteilungsmuster von ausschlaggebender Bedeutung ist ${ }^{18}$.

Es schien uns wünschenswert, die bisher erzielten Ergebnisse weiter zu vervollständigen, vor allem in Bezug auf den Einfluß der Applikationsweise und hinsichtlich der allmählichen Ausbildung des Verteilungsmusters im Anschluß an die Inkorporation sowie einer möglicherweise im Laufe der Zeit eintretenden Änderung in der Verteilung. Über diese speziellen Fragestellungen hinaus sind derartige Versuche - wie auch $\mathrm{C}_{\mathrm{ATSCH}}{ }^{13}$ a betont - geeignet, das allgemeine Verhalten von Metallkationen im Organismus weiter aufklären zu helfen.

\section{Untersuchungsmethodik}

Als Versuchstiere dienten 68 männliche und weibliche Meerschweinchen (Alter: 2-4 Monate, Gewicht: 300 bis $450 \mathrm{~g}$ ). Sie wurden während des Versuches gesondert gehalten und erhielten ständig eine gleichmäßig zusammengesetzte Standardkost.

9 E. H. Graul u. H. Hundeshagen, Atompraxis 2, 93 [1956].

10 B. Kawin, Nature [London] 179, 871 [1957].

11 K. E. Scheer, Strahlentherapie 101, 283 [1956].

12 D. Laszlo, Proc. Int. Conf. Atom. Energy 10, 62 [1956].

13 A. Catsch, a) Naturwissenschaften 43, 242 [1956]; b) Strahlentherapie 99, 290 [1956].
Bei dem verwendeten Isotop ${ }^{91} \mathrm{Y}$ handelt es sich um einen Beta-Gamma-Strahler $\left(E_{\beta \max }=1,6 \mathrm{MeV} ; E_{\gamma}=\right.$ $0,6 \mathrm{MeV}$ [Emissionswahrscheinlichkeit 0,03]) mit einer Halbwertszeit von 61 Tagen.

Injiziert wurden jeweils $10 \mu \mathrm{C}{ }^{91} \mathrm{Y}$, trägerfrei als Chlorid in 1,0 ml physiologischer NaCl-Lösung, $p_{\mathrm{H}} 3,5$. Dies entspricht einer Gewichtsdosis von $\approx 10^{-9} \mathrm{~g}$. Die Tiere wurden in 3 Gruppen eingeteilt: 1. intrakardiale, 2. intraperitoneale (rechter Unterbauch) und 3. subkutane Injektion (Rückenhaut in Thoraxhöhe). Die Tötung der Tiere erfolgte zu verschiedenen Zeiten nach der Injektion (3, 6 und $24 \mathrm{~h} ; 3,7$ und $14 \mathrm{~d})$ durch Übernarkotisieren mit Äther, wobei für jeden Zeitpunkt in jeder Gruppe mindestens 3 Tiere verwendet wurden.

Aktivitätshestimmungen wurden für folgende Organe durchgeführt: Blut, Herz, Lungen, Leber, Milz, Pankreas, Nieren, Nebennieren, Ovarien, Hoden und Femur (rechte Hinterextremität). Hierzu wurden die Organe in toto feucht verascht (konz. $\mathrm{HNO}_{3}$ ) und nach Eindampfen einer Probe auf dem Meßschälchen die Radioaktivität mit einem Glockenzählrohr gemessen. Eine Überprüfung zeigte gute Reproduzierbarkeit der auf diesem Wege ermittelten Meßwerte.

\section{Ergebnisse}

Die Aktivitäten der einzelnen Organe zu verschiedenen Zeiten nach der Inkorporation sind in den Abb. $1-5$ und der Tab. 1 angegeben. Außer den Werten in \% der injizierten Dosis findet sich auch ihre mittlere Streuung (mittlerer Fehler des Mittelwertes $\left.\mu= \pm \sqrt{\frac{\sum d^{2}}{n(n-1)}}\right)$ eingezeichnet. Sämtliche Werte sind entsprechend der Zerfallsrate korrigiert.

Intrakardial injiziertes trägerfreies ${ }^{91} \mathrm{Y}$ verschwindet relativ schnell aus der Blutbahn, nach $24 \mathrm{~h}$ werden noch $0,2 \%$ im Blut gefunden. Allerdings kommt es während der Beobachtungszeit nie zu völliger Aktivitätsfreiheit. Bei intraperitonealer und subkutaner Applikation werden die höchsten Werte innerhalb der ersten $6 \mathrm{~h}$ nach der Injektion beobachtet, wobei sich erwartungsgemäß die Resorption aus der Bauchhöhle als entschieden stärker erweist als aus dem subkutanen Gewebe. Messungen der Injektionsstelle in der Haut ließen noch nach $14 \mathrm{~d}$ hier den weitaus größten Teil der injizierten Dosis nachweisen.

14 J. G. Hamilton, Radiology 49, 325 [1947].

15 W. Bloom, Radiology 49, 344 [1947].

16 B. Rayner, M. Tutt u. J. Vaughan, Brit. J. exo. Pathol. 34, 138 [1953].

17 J. Vaughan, Brit. J. exp. Pathol. 36, 22 [1955].

18 E. Spode u. F. Gensicke, Naturwissenschaften 44, 118 [1957]. 


\begin{tabular}{|c|c|c|c|c|c|c|c|}
\hline Organ & $\begin{array}{l}\text { Applikations- } \\
\text { art }\end{array}$ & $3 \mathrm{~h}$ & $6 \mathrm{~h}$ & $24 \mathrm{~h}$ & $3 \mathrm{~d}$ & $7 \mathrm{~d}$ & $14 \mathrm{~d}$ \\
\hline Herz & $\begin{array}{l}\text { i.k. } \\
\text { i.p. } \\
\text { s.k. }\end{array}$ & $\begin{array}{l}1,0 \pm 0,33 \\
0,17 \pm 0,05 \\
0,05 \pm 0,01\end{array}$ & $\begin{array}{l}0,44 \pm 0,16 \\
0,10 \pm 0,07 \\
0,05 \pm 0,00\end{array}$ & $\begin{array}{l}0,44 \pm 0,20 \\
0,16 \pm 0,04 \\
0,04 \pm 0,01\end{array}$ & $\begin{array}{l}0,20 \pm 0,08 \\
0,10 \pm 0,01 \\
0,17 \pm 0,01\end{array}$ & $\begin{array}{l}0,27 \pm 0,04 \\
0,14 \pm 0,05 \\
0,08 \pm 0,01\end{array}$ & $\begin{array}{l}0,24 \pm 0,06 \\
0,12 \pm 0,01 \\
0,15 \pm 0,02\end{array}$ \\
\hline Lungen & $\begin{array}{l}\text { i.k. } \\
\text { i.p. } \\
\text { s.k. }\end{array}$ & $\begin{array}{l}1,86 \pm 0,60 \\
1,21 \pm 0,80 \\
0,28 \pm 0,15\end{array}$ & $\begin{array}{l}0,57 \pm 0,05 \\
0,35 \pm 0,14 \\
0,10 \pm 0,02\end{array}$ & $\begin{array}{l}0,56 \pm 0,12 \\
0,69 \pm 0,15 \\
0,45 \pm 0,08\end{array}$ & $\begin{array}{l}0,63 \pm 0,09 \\
0,40 \pm 0,01 \\
0,17 \pm 0,02\end{array}$ & $\begin{array}{l}0,65 \pm 0,08 \\
0,71 \pm 0,16 \\
0,90 \pm 0,40\end{array}$ & $\begin{array}{l}0,83 \pm 0,07 \\
0,38 \pm 0,02 \\
0,69 \pm 0,03\end{array}$ \\
\hline Pankreas & $\begin{array}{l}\text { i.k. } \\
\text { i.p. } \\
\text { s.k. }\end{array}$ & $\begin{array}{r}0,15 \pm 0,04 \\
22,15 \pm 16,15 \\
0,03 \pm 0,01\end{array}$ & $\begin{array}{l}0,07 \pm 0,03 \\
4,19 \pm 0,65 \\
0,03 \pm 0,01\end{array}$ & $\begin{array}{l}0,04 \pm 0,01 \\
8,05 \pm 0,51 \\
0,02 \pm 0,01\end{array}$ & $\begin{array}{r}0,07 \pm 0,01 \\
16,45 \pm 7,97 \\
0,04 \pm 0,02\end{array}$ & $\begin{array}{l}0,04 \pm 0,01 \\
1,26 \pm 0,36 \\
0,04 \pm 0,01\end{array}$ & $\begin{array}{l}0,03 \pm 0,01 \\
0,15 \pm 0,01 \\
0,03 \pm 0,00\end{array}$ \\
\hline $\begin{array}{l}\text { Neben- } \\
\text { nieren }\end{array}$ & $\begin{array}{l}\text { i.k. } \\
\text { i.p. } \\
\text { s.k. }\end{array}$ & $\begin{array}{l}0,11 \pm 0,08 \\
0,06 \pm 0,03 \\
0,01 \pm 0,01\end{array}$ & $\begin{array}{l}0,03 \pm 0,01 \\
0,05 \pm 0,01 \\
0,01 \pm 0,01\end{array}$ & $\begin{array}{l}0,02 \pm 0,00 \\
0,02 \pm 0,01 \\
0,02 \pm 0,01\end{array}$ & $\begin{array}{l}0,02 \pm 0,01 \\
0,05 \pm 0,01 \\
0,01 \pm 0,01\end{array}$ & $\begin{array}{l}0,03 \pm 0,01 \\
0,05 \pm 0,01 \\
0,03 \pm 0,01\end{array}$ & $\begin{array}{l}0,02 \pm 0,01 \\
0,02 \pm 0,01 \\
0,01 \pm 0,00\end{array}$ \\
\hline Ovarien & $\begin{array}{l}\text { i.k. } \\
\text { i.p. } \\
\text { s.k. }\end{array}$ & $\begin{array}{l}0,02 \pm 0,01 \\
0,01 \\
0,01 \pm 0,00\end{array}$ & $\begin{array}{l}0,02 \pm 0,01 \\
0,03 \pm 0,01 \\
0,00\end{array}$ & $\begin{array}{l}0,01 \pm 0,01 \\
0,00 \\
0,03\end{array}$ & $\begin{array}{l}0,03 \pm 0,02 \\
0,01 \\
0,02 \pm 0,01\end{array}$ & $\begin{array}{l}0,12 \pm 0,07 \\
0,02 \pm 0,01 \\
0,04\end{array}$ & $\begin{array}{l}0,03 \pm 0,00 \\
0,01 \\
0,02 \pm 0,01\end{array}$ \\
\hline Hoden & $\begin{array}{l}\text { i.k. } \\
\text { i.p. } \\
\text { s.k. }\end{array}$ & $\begin{array}{l}0,05 \\
0,15 \\
0,00\end{array}$ & $\begin{array}{l}0,07 \\
0,06 \\
0,02 \pm 0,01\end{array}$ & $\begin{array}{l}0,07 \\
0,10 \pm 0,02 \\
0,08\end{array}$ & $\begin{array}{l}0,04 \\
0,07 \pm 0,03 \\
0,03 \pm 0,01\end{array}$ & $\begin{array}{l}0,08 \\
0,08 \\
0,03 \pm 0,01\end{array}$ & $\begin{array}{l}0,08 \pm 0,00 \\
0,08 \pm 0,02 \\
0,01\end{array}$ \\
\hline
\end{tabular}

Tab. 1. Aktivität der Organe von Meerschweinchen nach Injektion von ${ }^{91} \mathrm{Y}(\%$ der injizierten Aktivität pro Organ) .

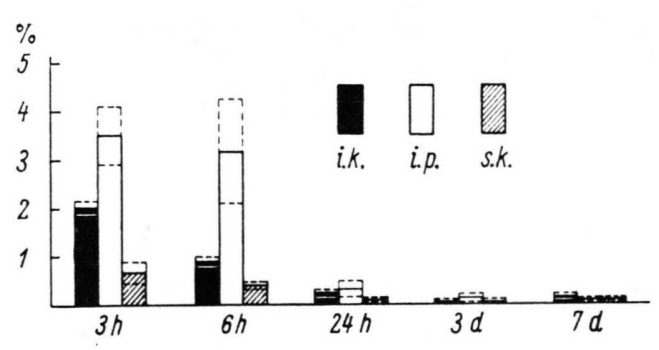

Abb. 1 Blutaktivität nach ${ }^{91}$ Y-Inkorporation.

Die Leberaktivität liegt besonders bei intrakardialer Injektion in der ersten Woche recht hoch, fällt aber langsam ab; nach $14 \mathrm{~d}$ wurden nur noch $3,5 \%$ in der Leber gefunden. Auch bei intraperitonealer Zuführung fallen die Leberwerte ab, erhöhen sich jedoch wieder, um dann ebenfalls beträchtlich abzusinken. Ein ähnliches Verhalten wird nach subkutaner Injektion beobachtet, wobei natürlich die Werte niedriger liecen.

Im Gegerssatz zur Leber wurde ${ }^{91} \mathrm{Y}$ von der Milz nur sehr geringgradig aufgenommen, wenn man von dem hohen 3-h-Wert bei intraperitonealer Applikation zunächst absieht. Die Aktivitäten erreichten maximal $0,6 \%$ der Injektionsdosis.

Die Nieren zeigten nach intrakardialer Injektion relativ hohe Anfangswerte, die im Laufe von $14 \mathrm{~d}$ deutlich zurückgingen. Die Intraperitoneal- und Sub-

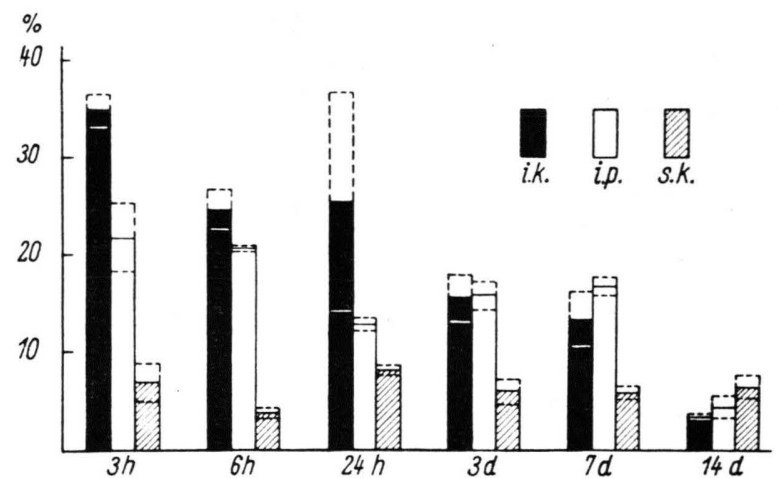

Abb. 2. Aktivität der Leber nach ${ }^{91}$ Y-Inkorporation.

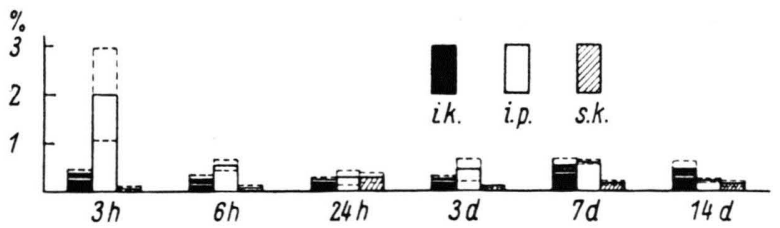

Abb. 3. Milzaktivität nach ${ }^{91}$ Y-Inkorporation.

kutan-Anfangswerte fielen zunächst gleichfalls ab. stiegen jedoch erneut an. um nach $14 \mathrm{~d}$ abzuklingen. Im ganzen zeigte sich eine Verlaufscharakteristik. die sich in guter Übereinstimmung mit dem Verhalten der Leberaktivitäten befindet. 


\begin{tabular}{|c|c|c|c|c|c|c|c|}
\hline Organ & $\begin{array}{c}\text { Applik.- } \\
\text { Art }\end{array}$ & $3 \mathrm{~h}$ & $6 \mathrm{~h}$ & $24 \mathrm{~h}$ & $3 \mathrm{~d}$ & 37 & $14 \mathrm{~d}$ \\
\hline Blut & $\begin{array}{l}\text { i.k. } \\
\text { i.p. } \\
\text { s.k. }\end{array}$ & $\begin{array}{l}0,88 \pm 0,16 \\
1,28 \pm 0,12 \\
0,26 \pm 0,02\end{array}$ & $\begin{array}{l}0,44 \pm 0,03 \\
1,42 \pm 0,43 \\
0,17 \pm 0,03\end{array}$ & $\begin{array}{l}0, \dot{0} 8 \pm 0,01 \\
0,43 \pm 0,15 \\
0,04 \pm 0,01\end{array}$ & $\begin{array}{l}0,03 \pm 0,02 \\
0,03 \pm 0,01 \\
0,03 \pm 0,02\end{array}$ & $\begin{array}{l}0,04 \pm 0,00 \\
0,02 \pm 0,00 \\
0,02 \pm 0,01\end{array}$ & $\begin{array}{l}0,03 \pm 0,01 \\
0,01 \pm 0,01 \\
0,01 \pm 0,01\end{array}$ \\
\hline Herz & $\begin{array}{l}\text { i.k. } \\
\text { i.p. } \\
\text { s.k. }\end{array}$ & $\begin{array}{l}7,66 \pm \quad 2,29 \\
1,21 \pm 0,29 \\
0,42 \pm 0,22\end{array}$ & $\begin{array}{l}4,16 \pm 1,84 \\
0,85 \pm 0,57 \\
0,39 \pm 0,03\end{array}$ & $\begin{array}{l}3,59 \pm 1,17 \\
1,39 \pm 0,09 \\
0,21 \pm 0,03\end{array}$ & $\begin{array}{l}1,54 \pm 0,36 \\
0,54 \pm 0,06 \\
0,42 \pm 0,06\end{array}$ & $\begin{array}{l}1,67 \pm 0,13 \\
0,80 \pm 0,40 \\
0,59 \pm 0,10\end{array}$ & $\begin{array}{l}1,74 \pm 0,38 \\
0,75 \pm 0,01 \\
0,94 \pm 0,15\end{array}$ \\
\hline Lungen & $\begin{array}{l}\text { i.k. } \\
\text { i.p. } \\
\text { s.k. }\end{array}$ & $\begin{array}{l}4,25 \pm 1,07 \\
3,60 \pm 2,16 \\
0,75 \pm 0,20\end{array}$ & $\begin{array}{l}2,38 \pm 0,38 \\
1,06 \pm 0,24 \\
0,33 \pm 0,08\end{array}$ & $\begin{array}{l}1,59 \pm 0,39 \\
1,92 \pm 0,93 \\
3,55 \pm 1,34\end{array}$ & $\begin{array}{l}2,27 \pm 0,49 \\
1,32 \pm 0,03 \\
1,15 \pm 0,16\end{array}$ & $\begin{array}{l}3,73 \pm 2,24 \\
1,55 \pm 0,39 \\
1,92 \pm 1,10\end{array}$ & $\begin{array}{l}2,62 \pm 0,60 \\
0,83 \pm 0,02 \\
1,68 \pm 0,21\end{array}$ \\
\hline Leber & $\begin{array}{l}\text { i.k. } \\
\text { i.p. } \\
\text { s.k. }\end{array}$ & $\begin{array}{r}18,38 \pm 2,86 \\
11,66 \pm 2,64 \\
3,54 \pm 0,20\end{array}$ & $\begin{array}{r}17,21 \pm 1,07 \\
13,17 \pm 3,09 \\
1,76 \pm 0,25\end{array}$ & $\begin{array}{l}8,39 \pm 4,11 \\
9,29 \pm 1,13 \\
4,97 \pm 0,41\end{array}$ & $\begin{array}{l}8,79 \pm 1,04 \\
7,21 \pm 1,14 \\
3,92 \pm 0,07\end{array}$ & $\begin{array}{l}6,31 \pm 1,26 \\
5,61 \pm 1,37 \\
3,99 \pm 0,60\end{array}$ & $\begin{array}{l}2,51 \pm 0,21 \\
1,92 \pm 0,91 \\
3,62 \pm 0,61\end{array}$ \\
\hline Milz & $\begin{array}{l}\text { i.k. } \\
\text { i.p. } \\
\text { s.k. }\end{array}$ & $\begin{array}{r}6,57 \pm 1,05 \\
37,39 \pm 29,81 \\
1,12 \pm 0,63\end{array}$ & $\begin{array}{l}5,00 \pm 0,68 \\
9,92 \pm 1,31 \\
1,39 \pm 0,33\end{array}$ & $\begin{array}{l}3,17 \pm 1,65 \\
4,03 \pm 1,30 \\
8,25 \pm 1,39\end{array}$ & $\begin{array}{l}4,88 \pm 1,08 \\
5,67 \pm 1,84 \\
2,16 \pm 0,10\end{array}$ & $\begin{array}{l}5,00 \pm 1,42 \\
4,17 \pm 0,40 \\
3,46 \pm 1,81\end{array}$ & $\begin{array}{l}4,50 \pm 1,14 \\
2,83 \pm 0,48 \\
2,20 \pm 0,88\end{array}$ \\
\hline Pankreas & $\begin{array}{l}\text { i.k. } \\
\text { i.p. } \\
\text { s.k. }\end{array}$ & $\begin{array}{r}1,14 \pm 0,25 \\
134,90 \pm 84,10 \\
0,20 \pm 0,03\end{array}$ & $\begin{array}{r}0,57 \pm 0,22 \\
36,35 \pm 7,65 \\
0,10 \pm 0,05\end{array}$ & $\begin{array}{r}0,30 \pm 0,15 \\
56,48 \pm 15,22 \\
0,08 \pm 0,03\end{array}$ & $\begin{array}{r}0,49 \pm 0,10 \\
106,00 \pm 33,90 \\
0,13 \pm 0,01\end{array}$ & $\begin{array}{l}0,23 \pm 0,02 \\
6,21 \pm 2,57 \\
0,33 \pm 0,08\end{array}$ & $\begin{array}{l}0,34 \pm 0,07 \\
1,21 \pm 0,02 \\
0,25 \pm 0,04\end{array}$ \\
\hline Nebenniere & $\begin{array}{l}\text { i.k. } \\
\text { i.p. } \\
\text { s.k. }\end{array}$ & $\begin{array}{l}4,03 \pm 2,40 \\
1,75 \pm 0,75 \\
0,18 \pm 0,03\end{array}$ & $\begin{array}{l}1,68 \pm 0,70 \\
3,25 \pm 1,18 \\
0,22 \pm 0,21\end{array}$ & $\begin{array}{l}0,85 \pm 0,23 \\
0,88 \pm 0,61 \\
0,33 \pm 0,26\end{array}$ & $\begin{array}{l}0,96 \pm 0,35 \\
1,87 \pm 0,09 \\
0,14 \pm 0,03\end{array}$ & $\begin{array}{l}0,89 \pm 0,12 \\
1,51 \pm 0,23 \\
1,04 \pm 0,08\end{array}$ & $\begin{array}{l}0,36 \pm 0,13 \\
1,06 \pm 0,05 \\
0,34 \pm 0,02\end{array}$ \\
\hline Nieren & $\begin{array}{l}\text { i.k. } \\
\text { i.p. } \\
\text { s.k. }\end{array}$ & $\begin{array}{r}30,48 \pm 5,52 \\
21,42 \pm 4,91 \\
5,60 \pm 0,92\end{array}$ & $\begin{array}{r}24,67 \pm 13,03 \\
9,92 \pm 1,92 \\
2,10 \pm 1,25\end{array}$ & $\begin{array}{r}15,51 \pm 3,54 \\
13,81 \pm 4,41 \\
3,41 \pm 1,04\end{array}$ & $\begin{array}{r}15,45 \pm 2,25 \\
8,05 \pm 1,95 \\
6,46 \pm 0,82\end{array}$ & $\begin{array}{l}8,88 \pm 2,29 \\
5,63 \pm 0,30 \\
4,69 \pm 0,84\end{array}$ & $\begin{array}{l}3,14 \pm 1,55 \\
1,81 \pm 0,35 \\
2,79 \pm 0,25\end{array}$ \\
\hline Ovarien & $\begin{array}{l}\text { i.k. } \\
\text { i.p. } \\
\text { s.k. }\end{array}$ & $\begin{array}{l}4,01 \pm 0,27 \\
2,65 \\
0,63 \pm 0,08\end{array}$ & $\begin{array}{l}2,94 \pm 0,18 \\
4,92 \pm 0,88 \\
0,52\end{array}$ & $\begin{array}{l}2,07 \pm 0,23 \\
0,38 \\
0,80\end{array}$ & $\begin{array}{l}5,90 \pm 0,37 \\
1,28 \\
1,15 \pm 0,09\end{array}$ & $\begin{array}{r}11,94 \pm 5,16 \\
1,39 \pm 0,68 \\
1,83\end{array}$ & $\begin{array}{l}3,08 \pm 0,58 \\
1,72 \\
1,62 \pm 0,53\end{array}$ \\
\hline Hoden & $\begin{array}{l}\text { i.k. } \\
\text { i.p. } \\
\text { s.k. }\end{array}$ & $\begin{array}{l}0,42 \\
0,98 \\
0,00\end{array}$ & $\begin{array}{l}0,80 \\
0,17 \\
0,10 \pm 0,02\end{array}$ & $\begin{array}{l}0,85 \\
0,89 \\
0,24\end{array} \pm 0,12$ & $\begin{array}{l}0,32 \\
0,35 \pm 0,07 \\
0,11 \pm 0,08\end{array}$ & $\begin{array}{l}0,79 \\
0,56 \\
0,20 \pm 0,05\end{array}$ & $\begin{array}{l}0,53 \pm 0,10 \\
0,26 \pm 0,12 \\
0,14\end{array}$ \\
\hline Femur & $\begin{array}{l}\text { i.k. } \\
\text { i.p. } \\
\text { s.k. }\end{array}$ & $\begin{array}{r}13,99 \pm 0,77 \\
7,46 \pm 0,79 \\
3,20 \pm 0,46\end{array}$ & $\begin{array}{r}14,32 \pm 3,32 \\
8,30 \pm 0,94 \\
3,35 \pm 0,72\end{array}$ & $\begin{array}{r}14,02 \pm 1,83 \\
16,17 \pm 7,11 \\
4,07 \pm 0,32\end{array}$ & $\begin{array}{r}14,20 \pm 1,20 \\
13,05 \pm 0,41 \\
7,45 \pm 1,73\end{array}$ & $\begin{array}{l}17,13 \pm 1,65 \\
13,87 \pm 0,38 \\
13,07 \pm 2,06\end{array}$ & $\begin{array}{l}19,16 \pm 2,42 \\
14,18 \pm 0,32 \\
13,09 \pm 0,67\end{array}$ \\
\hline
\end{tabular}

Tab. 2. Spezifische Aktivität der Organe von Meerschweinchen nach Injektion von ${ }^{91} \mathrm{Y}$ $(\%$ der injizierten Aktivität pro g Organ).

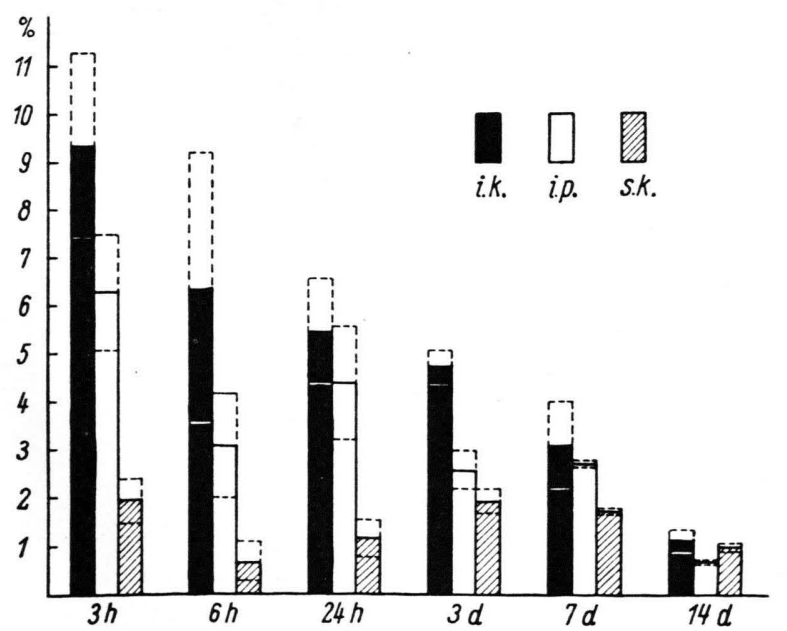

Abb. 4. Aktivität der Nieren nach ${ }^{91}$ Y-Inkorporation.
Auch bezüglich der Femuraktivität zeigten sich deutliche Unterschiede zwischen den einzelnen Applikationsarten. Nach intrakardialer Injektion betrug der 3-h-Wert 1,7 Prozent. Dieser Wert stieg kontinuierlich bis auf $2,4 \%$ nach $14 \mathrm{~d}$ an, was einer Speicherung im gesamten Skelettsystem von etwa 50\% entspricht. Bei den anderen Applikationsarten lagen die Werte, entsprechend den Resorptionsbedin-

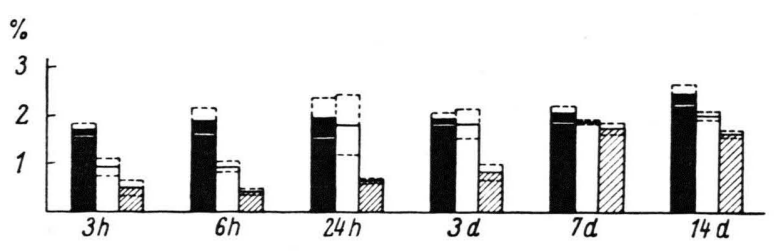

Abb. 5. Aktivität des Femur nach ${ }^{91}$ Y-Inkorporation. 
gungen zunächst relativ niedrig (i.p. $0,9 \%$, s.k. $0,5 \%$ ), doch stiegen sie - bei intraperitonealer Injektion schneller als bei subkutaner - ebenfalls an, erreich: ten aber innerhalb der Beobachtungszeit nicht den Intrakardialwert.

Herz und Lungen zeigten keine wesentlichen Aktivitäten, auch in den Nebennieren und den Geschlechts. organen wurden nur geringfügige Werte gefunden. Das Pankreas wies bei intrakardialer und subkutaner Injektion ebenfalls nur eine minimale Aktivität auf. Bemerkenswert dagegen sind die relativ sehr hohen Werte nach intraperitonealer Applikation, die wir schon bei unseren Mäuseversuchen (dort sogar noch ausgeprägter) beobachten konnten. Allerdings sind diese Aktivitäten mit einer großen Streuung belastet.

In der Tab. 2 (S. 289) sind die Werte der spezifischen Aktivität in $\%$ oo der injizierten Dosis pro g Organ aufgeführt. Diese Angaben sind deshalb von besonderem Interesse, weil die spezifische Aktivität ein Maß für die Strahlenbelastung des betreffenden Organs durch das inkorporierte Isotop darstellt.

Wir finden etwa die gleichen Verhältnisse wie bei der Betrachtung der $\%$-Werte pro Organ. Doch ergeben sich zusätzlich eine Reihe beachtenswerter Tatsachen. So liegt z. B. die spezifische Aktivität der Leber in den ersten $24 \mathrm{~h}$ bei jeder Applikationsart über den Femurwerten. Die spezifische Milzaktivität beträgt, abgesehen von einzelnen Intraperitonealwerten, nur etwa die Hälfte bis ein Drittel der Leberaktivitäten. Die höchsten Werte der spezifischen Aktivität während des ersten Tages weisen die Nieren auf, wenn die unten noch zu besprechenden intraperitonealen Pankreaswerte außer Betracht gelassen werden. Schließlich ist bemerkenswert. daß die spezifische Aktivität der Ovarien beträchtlich über der des Hodens liegt, was strahlenbiologisch von Bedeutung ist.

\section{Diskussion}

Die oben geschilderten Ergebnisse dürften geeignet sein, das bisherige Bild des Yttrium-Vertei. lungsmusters zu erweitern und abzurunden sowie auch methodische Hinweise für Verteilungsversuche allgemein zu geben.

Betrachten wir zunächst die Unterschiede. die sich bei den einzelnen Applikationsarten zeigen. so fällt vor allem nach intrakardialer Injektion die hohe initiale Leberaktivität auf. Das Abklingen der Leber- werte beruht zwar z. T. auf Ausscheidung des Yttriums durch die Galle in den Darm, wie eigene Untersuchungen zeigten, doch liegen diese Werte zu niedrig, um allein für den Aktivitätsverlust verantwortlich zu sein. Vielmehr scheint nach anfänglicher Leberspeicherung eine Mobilisierung zu erfolgen, die zu dem kontinuierlichen Anstieg der Y-Aktivität im Knochen mit beiträgt. Gleiche Vorgänge scheinen sich auch bei den anderen Applikationsarten abzuspielen, wenn sie auch nicht ganz so deutlich hervortreten.

Von Bedeutung scheint weiter die hohe Aktivitätsbelastung der Nieren. die sicher vorwiegend auf die Y-Ausscheidung im Harn zurückzuführen ist. Bei unseren Versuchen wurden innerhalb der ersten $24 \mathrm{~h}$ einige $\%$ der injizierten Dosis im Harn ausgeschieden (i.k.). Aber auch nach längerer Zeit, wenn im Harn praktisch keine Aktivität mehr gefunden wird bleibt eine Restaktivität in den Nieren zurück, die die Annahme einer echten Speicherung nahelegt.

Der Aktivitätsanstieg im Femur scheint am Schluf der Beobachtungszeit noch nicht beendet zu sein, wie uns einige 30-d-Werte zeigten. Bei getrennten Messungen fanden wir in den Epiphysen etwa die 5-fache Aktivität wie in der Diaphyse, während das Knochenmark die geringsten Werte aufwies. Dies entspricht gut den Ergebnissen von Bloom ${ }^{15}$.

Einer besonderen Besprechung bedarf die intraperitoneale Applikationsart. Die Aktivitäten sämtlicher Bauchorgane sind offenbar nur sehr schwierig zu beurteilen. Offensichtlich spielen - wie der Vergleich mit den anderen Applikationsarten zeigt Oberflächenaktivitäten (adsorptiv gebunden nach i.p. Injektion) eine große Rolle (z. B. bei Pankreas, Milz, wohl auch Leber). Typisch hierfür ist die hohe Pankreasaktivität, die sich nur bei intraperitonealer Injektion findet. Die Bauchspeicheldrüse liegt beim Meerschweinchen (auch bei der Maus!) ziemlich genau in der Injektionsrichtung relativ oberflächlich im Bereich der Duodenalschlinge unterhalb der groBen Kurvatur des Magens. Untersuchungen des Duodenums und des oberen Ileums zeigten auch hier erhebliche Aktivitäten. Die Resorptionsbedingungen sind dabei allerdings erheblich besser als aus dem Subkutangewebe, was auch von McDonald et coll. ${ }^{6}$ betont wird.

Die deutlichen Unterschiede. die sich bei verschiedenartiger Applikation hinsichtlich des Verteilungsmusters von Yttrium ergeben, dürften darauf beruhen. daß Yttrium als typisches Radiokolloid auch 
bei äußerst niedrigen Konzentrationen (trägerfrei!) schon ausgesprochen kolloidale Eigenschaften aufweist - s. hierzu $\mathrm{CATSCH}^{13}{ }^{13}$. Dies erklärt seine relativ hohe Affinität zu Organen des R.E.S., besonders der Leber, und seine schlechten Resorptionseigenschaften. Allerdings bleibt eine Reihe von Fragen noch offen, wie überhaupt das Problem des physikalisch-chemischen Zustandes der Radiokolloide im Körper und damit verbunden ihre Rolle im Stoffwechselgeschehen noch der Klärung harrt.

Ein sehr wesentliches Ergebnis der berichteten Versuche scheint uns zu sein, daß bei Verteilungsversuchen mit Spaltprodukten (dem Yttrium sehr ähnlich verhalten sich z. B. die Seltenen Erden) Befunde nach intraperitonealer Injektion mit äußerster Vorsicht zu betrachten sind, da hierbei Irrtümer auf- treten können, wie sie durch vorgetäuschte hohe Aktivitäten der Bauchorgane bedingt sind, die lediglich auf adsorptiver Anlagerung des Radioisotops an den Organoberflächen beruhen.

Schließlich scheint es uns wichtig, nochmals auf die Bedeutung der Leber als Passageorgan hinzuweisen. Die hohen Leberaktivitäten rechtfertigen es u. E., die Leber neben dem Knochensystem als kritisches Organ für Radioyttrium zu berücksichtigen. Auch die hohen Nierenwerte bedürfen sicher strahlenbiologischer Beachtung.

Herrn Prof. Dr. H.-J. Bons bin ich für die Anregung zu den Untersuchungen und für fördernde Diskussionen zu großem Dank verpflichtet. Fräulein E. W EBER danke ich für wertvolle Hilfe bei der technischen Durchführung der Versuche.

\title{
Uber ein polyploidisierendes Prinzip der Milz und seine quantitative biologische Bestimmung*
}

\author{
Von R. Rigler und W. Rosenkranz \\ Aus dem Physiologischen Institut der Universität Graz \\ (Vorstand Prof. Dr. R. Rigler) \\ (Z. Naturforschg. 13 b, 291-296 [1958]; eingegangen am 6. Februar 1958)
}

\begin{abstract}
An Ratten konnte gezeigt werden, daß die als Folge der Milzexstirpation im antimesometrialen Anteil eines traumatisch hervorgerufenen Deciduoms auf etwa das Doppelte pro Raumeinheit erhöhte Zahl an Deciduazellen durch subcutane Injektion eines wäßrigen Extraktes aus Ratten- oder Rindermilzen in Abhängigkeit von der Gabengröße gesenkı wird. Hierbei ließ sich ein Regressionskoeffizient gemäß einer nahezu linearen Abhängigkeit der Zahl der Deciduazellkerne vom Logarithmus des verwendeten Milzextrakt-Quantums errechnen. Die auf solche Weise gewonnenen Zahlenwerte erlauben Rückschlüsse auf die in Milzextrakten vorhandene Menge eines Stoffes mit vermutlich polyploidisierender Wirkung. Letztere zeigt sich außerdem in der Vergrößerung der Deciduakerne, worüber bereits an anderer Stelle berichtet wurde.
\end{abstract}

In mehreren vorausgehenden Mitteilungen ${ }^{1,2,3}$ wurde auf eine bisher unbekannte Korrelation hingewiesen, die zwischen dem lymphoreticulären Sy. stem, repräsentiert durch die Milz, und bestimmten mesenchymalen Wachstums- und Differenzierungsvorgängen besteht. Einen solchen stellt z. B. die Verwandlung der ruhenden Stromazelle des Endometriums in die Deciduazelle dar. Im künstlich gesetzten Deciduom der Ratte liegt nun ein Untersuchungsobjekt vor, das sich zur Prüfung verschiedener mit der Differenzierung und Zellvermehrung zusammen-

\footnotetext{
* Herrn Prof. Dr. C. L. Lautenschläger zum 70. Geburtstag gewidmet.

1 W. Rosenkranz, Ber. ges. Physiol. exp. Pharmakol. 180, 147 [1956].

2 R. Rigler, Wiener klin. Wschr. 68, 202 [1956].
}

hängender Fragen eignet., Die eingangs erwähnten Versuche hatten eine Abhängigkeit der Dichte der Deciduazell-Population von der Anwesenheit der Milz im Tierkörper erkennen lassen. Danach stellt bei der normalen Ratte die in der Raumeinheit eines bestimmten Gewebebereichs ermittelte DeciduazellZahl einen fast ebenso konstanten Wert wie die Zahl der roten Blutkörperchen im $\mathrm{mm}^{3}$ dar. Über weitere gleichartige Ergebnisse wird unten berichtet.

Es wurde ferner beobachtet ${ }^{4}$, daß die Milz auch einen Einfluß auf die Kerngröße dieser Zellen aus-

3 R. Rigler u. W. Rosenkranz, Wiener klin. Wschr. 69, 445 [1957].

4 W. Rosenkranz u. R. Rigler. Z. ges. exp. Med. 129, 627 [1958]. 